\title{
Development and Evaluation of a Highly Reliable Assay for SUMO-Specific Protease Inhibitors
}

\author{
Wenjuan Xie, Zhongli Wang, Jianchen Zhang, Lie Wang, Yaxue Zhao, Huchen Zhou* \\ School of Pharmacy, Shanghai Jiao Tong University, Shanghai 200240, China
}

ARTICLE INFO

\section{Article history:}

Received

Revised

Accepted

Available online

\section{Keywords:}

SUMO

SENP

deSUMOlyation

Inhibitor

Assay
ABSTRACT

SUMOylation, as a post-translational modification of proteins, plays essential regulatory roles in a variety of pathological conditions. In the dynamic process of SUMOylation and deSUMOylation, SENPs (SUMO-specific proteases), in charge of deconjugation of SUMO (small ubiquitin-related modifier) from substrate proteins, have recently been found to be potential therapeutic targets for cancer treatment. A reliable and practical assay is much needed to accelerate the discovery of SENPs inhibitors. We established a quantitative assay based on readily available SDS-PAGE-Coomassie system using RanGAP-SUMO as the substrate, thus avoiding the use of expensive fluorescent dyes or the error-prone fluorescent reporter. Its reproducibility and reliability were also evaluated in this report.
SUMOylation, as a post-translational modification of substrate proteins, has been shown to regulate a vast array of diverse cellular processes, such as transcription, DNA repair, signal transduction, and cell cycle. ${ }^{1-3}$ Modification of proteins by SUMO is a dynamic and reversible process which is controlled by an enzymatic pathway. ${ }^{4}$ SUMOylation involves the covalent attachment of SUMO to the $\varepsilon$-amino group of lysine residues in specific target proteins through an enzymatic cascade that requires a sequential action of E1 activating enzymes, E2 conjugating enzymes, and E3 ligases. ${ }^{5,6}$ On the other hand, the deSUMOylation is catalyzed by a family of SENPs (SUMOspecific proteases). In mammalian cells, there has been reported six isoforms of SENPs (SENP1, SENP2, SENP3, SENP5, SENP6 and SENP7), which differ in their sequence homology, substrate specificity, and subcellular localization. ${ }^{7-9}$ The isopeptidase activity of SENPs is essential for the recycling of SUMOylated substrate, and has been shown to be implicated in various cancers including prostate cancer, ${ }^{10,11}$ thyroid cancer, ${ }^{12}$ colon cancer, ${ }^{13}$ lung cancer, ${ }^{14}$ atherosclerosis, ${ }^{15}$ and heart disease. $^{16}$ Discovery of potent SENPs inhibitors, especially isoform-selective inhibitors, will facilitate the probing of SENPs' physiological roles and the validation of them as novel therapeutic targets.

However, to this date, very few small molecule SENPs inhibitors have been reported and their potency needs significant improvement. ${ }^{17-22}$ Furthermore, development of isoformselective inhibitors remained a highly challenging task. When we embarked on this task, we found that the existing assay methods for SENPs inhibitors limited the discovery process, due to low reliability and high cost. In earlier studies, researchers used SUMO-AMC as a substrate so that the cleavage of the substrate can be followed by spectrofluorometric readout. ${ }^{23}$ In another method, GFP and YFP are fused to both ends of substrate so that the FRET signal can be followed for measurement. ${ }^{24,25} \mathrm{~A}$ SUMO-CHOP-Reporter assay was developed using SUMO$\mathrm{PLA}_{2}$ fusion protein as the substrate, following the release of free $\mathrm{PLA}_{2}$ which converts its own substrate to a fluorescent product. ${ }^{26}$ These methods all require modification of the natural substrate, thus may not represent the actual cleavage of the natural substrate. Furthermore, the preparation of the fusion proteins require cumbersome conjugation and purification steps. Although the SUMO-CHOP-Reporter kit is commercially available, it proved labile to degradation during handling and shipment, thus frequently failing to give reproducible results based on experience of researchers. Besides, this assay may give false positive results due to unexpected inhibition of the PLA $\mathrm{PL}_{2}$ by test compounds. At the same time, fluorescence measurement in solution is known for its tendency to be influenced by various experimental factors and may cause confusion when structureactivity relationship (SAR) is closely analyzed. Furthermore, the reliability and robustness of these methods have never been assessed in practical applications.

Thus, although these methods are useful for high-throughput screening, a more reliable and practical quantitative assay is much needed in a robust structural optimization effort in order to reliably decipher SAR and discover potent and isoform-selective

*Corresponding author. Tel.: +86-21-3420-6721; e-mail : hczhou@ @jtu.edu.cn. 
inhibitors. Natural substrates of enzymes are often used for the initial characterization of the activity of the recombinant enzymes by following the cleavage of substrates on SDS-PAGE using fluorescent dyes. ${ }^{5}$ We decided to develop a practical and quantitative percentage inhibition and $\mathrm{IC}_{50}$ assays based on the cleavage of the natural substrate. Here we describe the establishment and evaluation of a robust assay method based on readily available SDS-PAGE-Coomassie ${ }^{27}$ system and RanGAPSUMO as the substrate protein. We believe it will facilitate the future efforts to discover potent and selective SENPs inhibitors.

The catalytic core of human SENP1 (residues 419-644) (SENP1-cc) was cloned into pET28a vector for expression as a His-tagged protein from E. coli strain BL21 (DE3). The protein was purified by nickel affinity chromatography at a final yield of $4.56 \mathrm{~g}$ per liter, while SENP2 (amino acids 363-589) (SENP2-cc) was obtained at a final yield of $3.42 \mathrm{~g}$ per liter (Figure 1). To prepare the substrate RanGAP-SUMO, the plasmids pET28aRanGAP and PT-SAE1/SAE2-SUMO were co-transformed into the E.coli strain BL21 (DE3), and the protein was purified by nickel affinity chromatography followed by anion exchange chromatography Mono-Q subsequently to obtain RanGAPSUMO in high purity. ${ }^{28}$

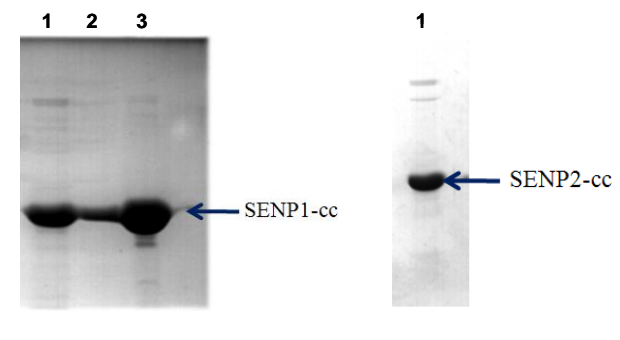

Figure 1. Purified His-tagged SENP1-cc and SENP2-cc.

As the first step to establish the assay, the optimal concentration of SENP needs to be determined in order to ensure that the enzyme concentration is in linear relationship to its catalytic activity. The deSUMOylation reaction was carried out by incubating excessive amount of RanGAP-SUMO $(10 \mu \mathrm{g})$ with SENP1-cc at concentrations varying from 0.2 to $8.0 \mathrm{nM}$. The reactions were terminated by addition of SDS loading buffer and analyzed on SDS-PAGE gel after Coomassie blue staining. The RanGAP-SUMO bands were quantified using the Odyssey infrared scanner (excitation $680 \mathrm{~nm}$, emission $720 \mathrm{~nm}$ ), and the percentage of hydrolyzed RanGAP-SUMO was calculated as $\left(\mathrm{F}_{0}-\mathrm{F}_{\mathrm{n}}\right) / \mathrm{F}_{0}$, whereas $\mathrm{F}_{0}$ is the initial RanGAP-SUMO and $\mathrm{F}_{\mathrm{n}}$ is the remaining RanGAP-SUMO. This resulted in the enzyme concentration-activity curve as shown in Figure 2A. The enzyme activity is linear to its concentration below $1 \mathrm{nM}$, thus enzyme concentration of $0.8 \mathrm{nM}$ whereas $65 \%$ substrate RanGAP-SUMO was cleaved was determined as the SENP1 concentration in the following assays. To ensure future batches of enzyme will give consistent test results, we defined 1 U SENP1 as the concentration which will result in $65 \%$ of the substrate cleaved, and 1 U SENP1 will be consistently used in all tests.

Next, the time-activity curve was obtained over the time course of 0 to 60 minutes as shown in Figure 2B. Based on this curve, a reaction time of 25 minutes was chosen to be used in the assay as it was near completion of substrate cleavage without reaching the plateau. The assay condition of SENP2 was similarly determined to be $6 \mathrm{nM}$ and $30 \mathrm{~min}$, and $1 \mathrm{U}$ SENP2 was defined as the concentration which results in $50 \%$ of the substrate cleaved.

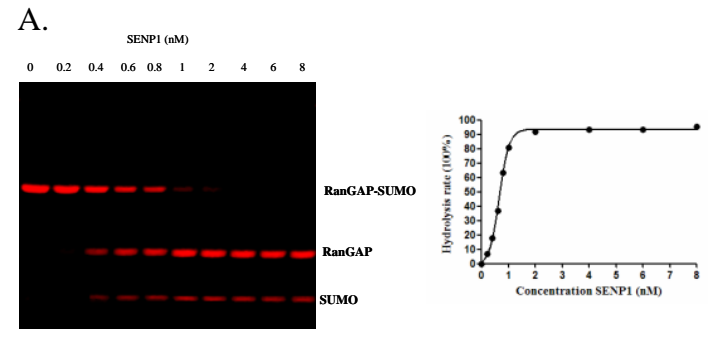

B.

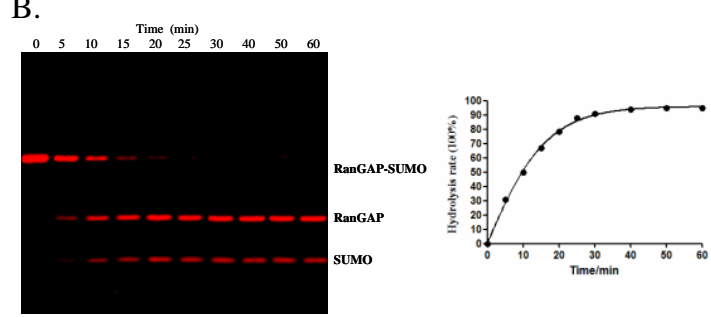

Figure 2. A. The deSUMOylation of RanGAP-SUMO at different concentrations of SENP1, reaction time $15 \mathrm{~min}$, at $37{ }^{\circ} \mathrm{C}$ (left); the resulting concentration curve (right). B. SENP1 deSUMOylation of RanGAP-SUMO at different reaction time points (0-60 min), SENP1 at $1 \mathrm{U}$ (left); the resulting time curve (right). Gels were stained with Coomassie blue and scanned on the Odyssey infrared scanner.

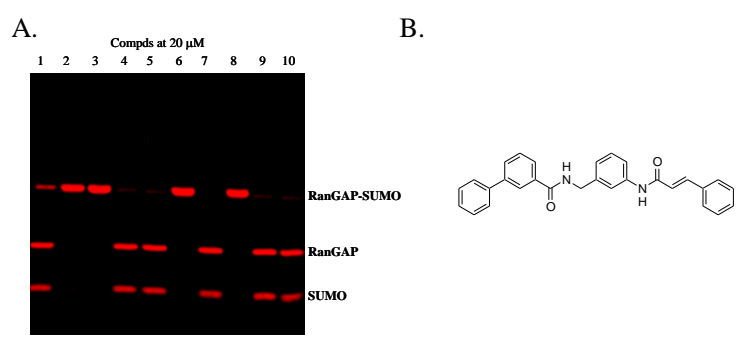

Figure 3. (A) Screening of ten compounds for their SENP1 inhibitory activity. SENP1: $1 \mathrm{U}$; reaction time: $25 \mathrm{~min}$; RanGAP-SUMO: $10 \mu \mathrm{g}$. (B) Chemical structure of compound $\mathbf{3}$ as a representative structure of the inhibitors whose structure-activity relationship will be published elsewere.

Thus, for the initial screening of inhibitors, 1 U SENP1 was incubated with compounds at $20 \mu \mathrm{M}$ for $10 \mathrm{~min}$ before $10 \mu \mathrm{g}$ RanGAP-SUMO was added, and incubated for another $25 \mathrm{~min}$. After termination of the reaction by addition of SDS loading buffer, the samples were subjected to SDS-PAGE gel electrophoresis, stained with Coomassie blue, and scanned using the Odyssey infrared scanner. ${ }^{29,30}$ Compounds $2,3,6$, and $\mathbf{8}$ showed good inhibitory activities (Figure 3). For $\mathrm{IC}_{50}$ measurement, compound $\mathbf{3}$ at interval concentrations was tested (Figure 4A) and the percentage of hydrolyzed RanGAP-SUMO was calculated as $\left(\mathrm{F}_{0}-\mathrm{F}_{\mathrm{n}}\right) / \mathrm{F}_{0}$, whereas $\mathrm{F}_{0}$ is the initial RanGAPSUMO and $F_{n}$ is the remaining RanGAP-SUMO. The data was fitted with GraphPad to give the $\mathrm{IC}_{50}$ value (Figure $4 \mathrm{~A}$ ). In structure-activity relationship studies, it is critical that $\mathrm{IC}_{50}$ measurement is reliably consistent during the study. Thus, we evaluated the consistency and stability of our assay method by repeating the measurement three times independently during a period of three days. The result in Figure $4 \mathrm{~A}$ showed the $\mathrm{IC}_{50}$ measurement is highly consistent, i.e. 3.6, 3.8, and 3.3 $\mu \mathrm{M}$. The same assay was carried out with SENP2 in a similar manner, and the assay was also shown to be highly reproducible (Figure 4B). 

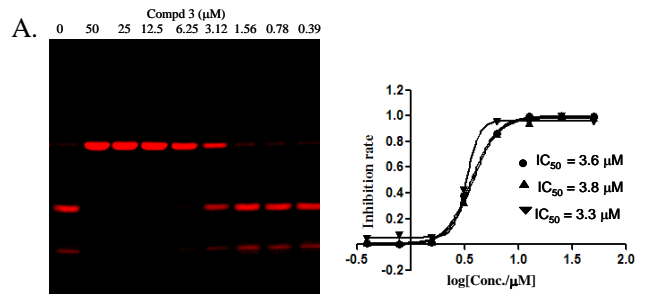

B.
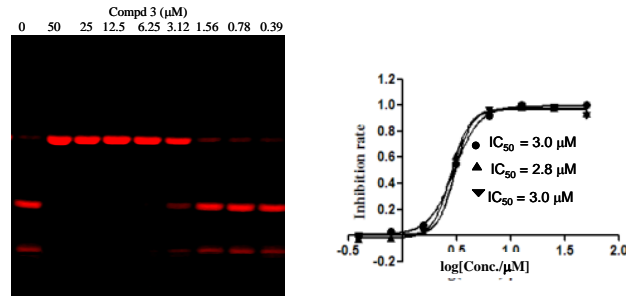

Figure 4. The $\mathrm{IC}_{50}$ measurement of compound 3. (A) Inhibition of SENP1, SENP1 $1 \mathrm{U}$, reaction time $25 \mathrm{~min}$; (B) Inhibition of SENP2, SENP2 $1 \mathrm{U}$, reaction time $30 \mathrm{~min}$. Gels were stained with Coomassie blue and scanned on the Odyssey infrared scanner. $\mathrm{IC}_{50}$ was measured three times independently during a period of three days to evaluate its stability, error bars were omitted for clarity of presentation.

Fluorescent dyes, such as Krypton protein stain, are often used when quantification of protein is needed. In order to compare the effect of Coomassie blue-based quantification to fluorescent dyes, we carried out the deSUMOylation assay using Krypton $^{30}$ instead of Coomassie. As shown in Figure 5A and 5B, the $\mathrm{IC}_{50}$ data and its reproducibility are almost identical to those from Coomassie blue staining. Thus, the use of readily available Coomassie blue staining in this assay gave quality data as good as expensive and specialized fluorescent dye, and the staining procedure proved to be significantly faster $(50 \mathrm{~min} v s .160 \mathrm{~min})$.
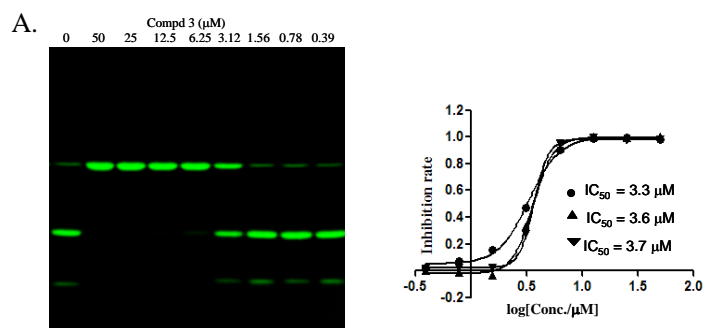

B.
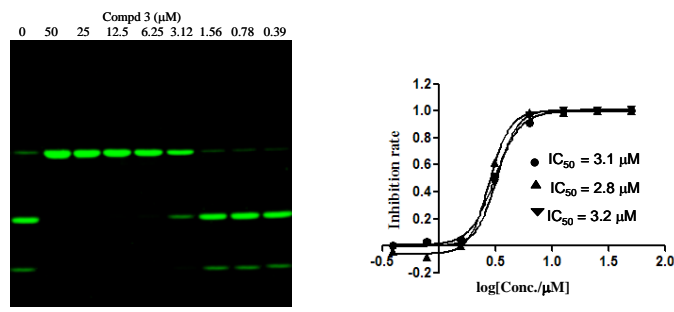

Figure 5. The $\mathrm{IC}_{50}$ measurement of compound 3. (A) Inhibition of SENP1, SENP1 $1 \mathrm{U}$, reaction time $25 \mathrm{~min}$; (B) Inhibition of SENP2, SENP2 $1 \mathrm{U}$, reaction time $30 \mathrm{~min}$. Gels were stained with Krypton fluorescent dye and scanned on the FLA-5100 imaging system. IC $_{50}$ was measured three times independently during a period of three days to evaluate its stability, error bars were omitted for clarity of presentation.

As a novel and potential therapeutic target in cancer treatment, SENPs have recently drawn much attention. Discovery of potent and isoform-selective SENPs inhibitors are imperative in understanding the complex physiological roles of SENPs and eventually discovery of clinically useful compounds.
Thus, a practical and reliable assay is necessary for future indepth effort in the search of SENPs inhibitors. Here, we developed a highly reproducible and readily available assay based on hydrolysis of RanGAP-SUMO and Coomassie blue staining. While this assay was not designed as a high-throughput screening method, it serves as a reliable quantitative measurement thus useful when close examination of SAR is needed at the structural optimization stage. Furthermore, it can be successfully applied to different isoforms of SENPs. We believe it will significantly facilitate the development of potent and isoform-selective SENPs inhibitors in the future.

\section{Acknowledgements}

We thank National Science Foundation of China (81222042; 81573264), Ministry of Science and Technology of China (2010CB912104), E-Institutes of Shanghai Universities (EISU) Chemical Biology Division, and Shanghai Jiao Tong University New Faculty Start Fund (14X100040051) for financial support of this work. The expression plasmids SENP1, SENP2, RanGAP, and SUMO were kindly provided by Prof. Yingli Wu (SJTU).

\section{References and notes}

1. Hochstrasser, M. Nature 2009, 458, 422

2. Heun, P. Curr. Opin. Cell Biol. 2007, 19, 350.

3. Bettermann, K.; Benesch, M.; Weis, S.; Haybarck, J. Cancer Lett., 2012, 316, 113.

4. Yeh, E. T. J. Biol. Chem. 2009, 284, 8223

5. Lois, L. M.; Lima, C. D. EMBO J. 2005, 24, 439.

6. Tang, Z. S.; Hecker, C. M.; Scheschonka, A.; Betz, H. FEBS J. 2008, 275, 3003.

7. Mikolajczyk, J.; Drag, M.; Bekes, M.; Cao, J. T.; Ronai, Z.; Slavesen, G. S. J. Biol. Chem. 2007, 282, 26217.

8. Hickey, C. M.; Wilson, N. R.; Hochstrasser, M. Nat. Rev. Mol. Cell Biol. 2012, 13, 755 .

9. Kim, J. H.; Baek, S. H. Biochim. Biophys. Acta 2009, 1792, 155.

10. Cheng, J.; Bawa, T.; Lee, P.; Gong, L.; Yeh, E. T. Neoplasia 2006, 8, 667.

11. Wang, Q.; Xia, N.; Li, T.; Xu, Y.; Zou, Y.; Fan, Q.; Khalfe, T. B.; Yeh, E. T.; Cheng, J. Oncogene 2013, 32, 2493.

12. Jacques, C.; Baris, O.; Prunier-Mirebeau, D.; Savagner, F.; Rodien, P.; Rohmer, V.; Franc, B.; Guyetant, S.; Malthiery, Y.; Reynier, P. J. Clin. Endocrinol. Metab. 2005, 90, 2314.

13. Xu, Y.; Li, J.; Zuo, Y.; Deng, J.; Wang, L. S.; Chen, G. Q. Cancer Lett. 2011, 309, 78 .

14. Wang, R. T.; Zhi, X. Y.; Zhang, Y.; Zhang, J. Exp. Ther. Med. 2013, 6,1054

15. Heo, K. S.; Chang, E.; Le, N. T.; Cushman, H.; Yeh, E. T.; Fujiwara, K.; Abe, J. Circ. Res. 2013, 112, 911.

16. Kim, E. Y.; Chen, L.; Ma, Y.; Yu, W.; Chang, J.; Moskowitz, I. P.; Wang, J. J. Mol. Cell. Cardiol. 2012, 52, 638.

17. Qiao, Z. T.; Wang, W. W.; Wang, L.; Wen, D. H.; Zhao, Y. X.; Wang, Q.; Meng, Q. Q.; Chen, G. Q.; Wu, Y. L.; Zhou, H. C. Bioorg. Med. Chem. Lett. 2011, 21, 6389.

18. Madu, I. G.; Namanja, A. T.; Su, Y.; Wong, S.; Li, Y. J.; Chen, Y. ACS Chem. Biol. 2013, 8, 1435.

19. Albrow, V.; Ponder E.; Fasci, D.; Bekes, M.; Deu, E.; Salvesen, G.; Bogyo, M. Chem. Biol. 2011, 18, 722.

20. Uno, M.; Koma, Y.; Ban, H. S.; Nakamura, H. Med. Chem. Lett. 2012, 22, 5169 .

21. Kumar, A.; Ito, A.; Takemoto, M.; Yoshida, M.; Zhang, K. Y. J. Chem. Inf. Model. 2014, 54, 870 .

22. Sommer, S.; Weikart, N. D.; Linne, U.; Mootz, H. D. Bioorg. Med. Chem. 2013, 21, 2511.

23. Wilkinson, K. D.; Gan-Erdene, T.; Kolli, N. Methods Enzymol. 2005, 399, 37

24. Tatham, M. H.; Hay, R. T. Methods Mol. Biol. 2009, 497, 253.

25. Rouleau, N.; Wang, J. H.; Karras, L.; Andrew, E.; BielefeldSevigny, M.; Chen, Y. Anal. Biochem. 2008, 375, 364.

26. Nicholson, B.; Leach, C. A.; Goldenberg, S. J.; Francis, D. M.; Kodrasov, M. P.; Tian, X.; Shanks, J.; Sterner, D. E.; Bernal, A.; Mattern, M. R.; Wilkinson, K. D.; Butt, T. R. Protein Sci. 2008, 17,1035 . 
27. Luo, S.; Wehr, N. B.; Levine, R. L. Anal. Biochem. 2006, 15, 233.

28. Expression and purification of RanGAP-SUMO: The plasmids pET28a-RanGAP and PT-SAE1/SAE2-SUMO were cotransformed into the E. coli strain BL21 (DE3), which was screened with kanamycin and chloramphenicol. Expression of the recombinant protein was induced with $1 \mathrm{mM} \mathrm{IPTG}$ at $27{ }^{\circ} \mathrm{C}$ for 14 h. The bacteria were isolated by centrifugation at $4500 \mathrm{rpm}$ for 10 min and the obtained pallet was resuspended in lysis buffer (50 $\mathrm{mM} \mathrm{Na} 2 \mathrm{HPO}_{4} / \mathrm{NaH}_{2} \mathrm{PO}_{4}$ (pH 7.4), $300 \mathrm{mM} \mathrm{NaCl}, 10 \%$ glycerol, $10 \mathrm{mM} \beta$-mercaptoethanol, and $10 \mathrm{mM}$ imidazole) and ruptured by sonication. Cell free extract was bound to Ni-NTA resin (Qiagen) and washed extensively with lysis buffer. The protein was eluted with lysis buffer containing $150 \mathrm{mM}$ imidazole, then further purified using anion exchange chromatography (Mono-Q, $20 \mathrm{mM}$ Tris- $\mathrm{HCl}$ to $20 \mathrm{mM}$ Tris- $\mathrm{HCl}+1 \mathrm{M} \mathrm{NaCl}$ ). The eluted RanGAP-SUMO was concentrated with ultracentrifugation tube (Milipore).

29. DeSUMOylation assay: 1 U SENP1 was incubated with compounds at $20 \mu \mathrm{M}$ at $37^{\circ} \mathrm{C}$ for $10 \mathrm{~min}$ in the deSUMOylation reaction buffer $(50 \mathrm{mM}$ Tris- $\mathrm{HCl} \mathrm{pH} 8.0,20 \mathrm{mM} \mathrm{NaCl}, 2 \mathrm{mM}$ DTT, $2 \mathrm{mM} \mathrm{CaCl})_{2}$. After $5 \mu \mathrm{L}$ RanGAP-SUMO $(2 \mu \mathrm{g} / \mu \mathrm{L})$ was added and incubated at $37^{\circ} \mathrm{C}$ for $25 \mathrm{~min}$, the reaction was terminated by addition of SDS loading buffer. The samples were subjected to SDS-PAGE and Coomassie blue staining. The RanGAP-SUMO bands were quantified on the Odyssey infrared scanner $\left(\lambda_{\mathrm{ex}}=680 \mathrm{~nm}\right.$ and $\left.\lambda_{\mathrm{em}}=720 \mathrm{~nm}\right)$. Percentage inhibition was calculated using the following equation: \%inhibition = $\left[\left(\mathrm{RFU}_{\text {sample }}-\mathrm{RFU}_{\text {blank }}\right) /\left(\mathrm{RFU}_{\text {substrate }}-\mathrm{RFU}_{\text {blank }}\right)\right]$ x $100 \%$, sample RanGAP-SUMO, SENP1, and compounds in assay buffer, blank RanGAP-SUMO, SENP1 in assay buffer, substrate - RanGAPSUMO in assay buffer without SENP1. For $\mathrm{IC}_{50}$ measurement, a test compound at interval concentrations $(50,25,12.5,6.25,3.12$, $1.56,0.78,0.39 \mu \mathrm{M})$ was incubated with $1 \mathrm{U}$ SENP1 at $37^{\circ} \mathrm{C}$ for $10 \mathrm{~min}$ in reaction buffer before $5 \mu \mathrm{L}$ RanGAP-SUMO $(2 \mu \mathrm{g} / \mu \mathrm{L})$ was added, and incubated at $37{ }^{\circ} \mathrm{C}$ for another $25 \mathrm{~min}$. The samples were treated in a manner similar to the percentage inhibition measurement, and the data was fitted with GraphPad to give the $\mathrm{IC}_{50}$ value. SENP2 inhibitors were assayed in a similar manner at $1 \mathrm{U}$ enzyme and $30 \mathrm{~min}$ reaction time.

30. Gel staining: For Coomassie blue staining, gel was rinsed with water, stained for $10 \mathrm{~min}$ with $0.01 \%$ Coomassie blue R250 (Sigma) in 50\% methanol (v/v) and $10 \%$ acetic acid $(\mathrm{v} / \mathrm{v})$, rinsed with $40 \%$ methanol (v/v) and $7 \%$ acetic acid (v/v) followed by destaining for $30 \mathrm{~min}$ in the same solution, and soaked twice in water, 5 min each time. Gels were scanned on the Odyssey infrared scanner $\left(\lambda_{\mathrm{ex}}=680 \mathrm{~nm}\right.$ and $\left.\lambda_{\mathrm{em}}=720 \mathrm{~nm}\right)$. For Krypton fluorescent staining, gel was fixed twice with a solution containing $40 \%$ ethanol (v/v) and 10\% (v/v) acetic acid, 30 min each time. After rinsed with water for $5 \mathrm{~min}$, it was stained with 1x Krypton protein stain for $1 \mathrm{~h}$, rinsed with $5 \%(\mathrm{v} / \mathrm{v})$ acetic acid followed by destaining for $5 \mathrm{~min}$ in the same solution, soaked twice in water, 15 min each time. Gels were scanned on the FLA5100 imaging system $\left(\lambda_{\mathrm{ex}}=532 \mathrm{~nm}\right.$ and $\left.\lambda_{\mathrm{em}}=580 \mathrm{~nm}\right)$. 


\section{Graphical Abstract}

Development and Evaluation of a Highly

Leave this area blank for abstract info.

Reliable Assay for SUMO-Specific Protease

Inhibitors

Wenjuan Xie, Zhongli Wang, Jianchen Zhang, Lie Wang, Yaxue Zhao, Huchen Zhou
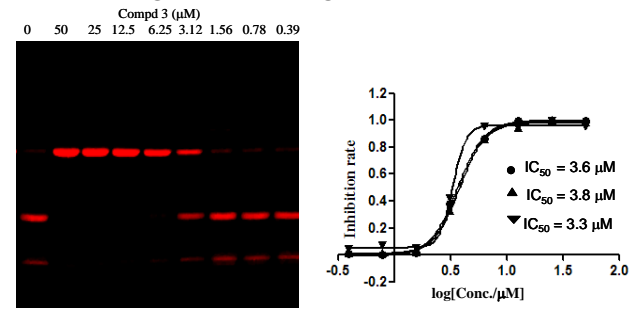